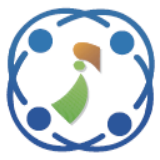

\title{
Enhanced Voice Service (EVS) Codec Using TCP Reno in Voice over Internet Protocol in LTE Network
}

\author{
Smita Avinash Lonkar ${ }^{1 *}$ \\ Kuraparthi Tirumala Viswanatha Reddy ${ }^{2}$ \\ ${ }^{1}$ Usha Mittal Institute of Technology, India \\ ${ }^{2}$ Sir Visvesvaraya Institute of Technology, India \\ * Corresponding author’s Email: smitalonkar@gmail.com
}

\begin{abstract}
Voice over Internet protocol (VoIP) is one of the online streaming method to provide voice communication that is used in the organization voice operation or conference call. In recent years, researcher developed several methods for improving the voice quality; but it still faces some problems of high jitter, delay and low throughput. In this paper, a VoIP is introduced to enable voice calls on Long Term Evolution (LTE). The Enhanced Voice Service (EVS) - Transmission Control Protocol (TCP) Reno-VoIP (EVS-TCP Reno-VoIP) method is introduced for enhancing the voice quality on LTE network. The EVS codec is used for improving the voice service and TCP Reno is used to avoid voice congestion in the LTE network. The testbed experiments on voice based skype application is implemented for LTE network. The network traffic is computed using the Jperf software. The Quality of Service (QoS) of the VoIP over LTE is analysed in Network Simulator 2 (NS2). The multiple scenarios were simulated to compute and analyse the performances in terms of an end to end delay, throughput, jitter and packet loss. The simulation results showed that EVS-TCP Reno-VoIP method has improved 2-3.5\% of network performance compared to existing methods such as EVS codec, G-11, G-723 and SDSS.
\end{abstract}

Keywords: Enhanced voice service, Long term evolution, Network simulator, Third generation, Transmission control protocol Reno, Quality of service, Voice over Internet protocol.

\section{Introduction}

The growth of the smartphone traffic has concentrated on performance improvements in an Enhanced - up - Link (EUL) scheduling in the Wideband Code Division Multiple Access (WCDMA) cellular system [1]. Laterally, the analog cellular has been replaced by digital cellular via 2Generation (2G) network that provides better capacity, enhanced quality and an upgraded security. It is analyzed that the mobile networking can accommodate web-based applications like video, audio files and it can transmit data rates of up to $384 \mathrm{kbit} / \mathrm{s}$ via $3 \mathrm{G}[2,3]$. The LTE network is used to generate air interface by $3^{\text {rd }}$ Generation Partnership Project (3GPP). Initially, the 3G WCDMA has provided a high capacity and air interface including transportation of packet traffic. The radio access network is designed to be compatible with the second generation Global System Mobile (GSM) and General Packet Radio Services (GPRS) network [4].

The $3 \mathrm{G}$ and $4 \mathrm{G}$ networks are more suitable for voice conferencing call because of $384 \mathrm{~kb} / \mathrm{s}$ of data transfer rates. The VoIP technique helps to transmit real-time voice over a Packet Switched Networks (PSNs) [5, 6]. The VoIP has improved the communication because it provides a number of other facilities like files, data, screen sharing and especially video chats $[7,8]$. Developing a VoIP quality prediction model for mobile users is essential to monitor and plan networks for a better QoS as well as better quality of experience to manage customers $[9,10]$. In this research work, the EVS codec is used for better voice services. The TCP Reno protocol is used to avoid the congestion in the network, which uses the slow start threshold to distinguish the phase of a slow start and the phase 
of the congestion avoidance. The data are collected from test bed experiments on voice skype applications. The network traffic is analyzed by employing the Jperf software tool to determine the network performance and measurement. The experimental result computes the performance of EVS-TCP Reno-VoIP in terms of throughput, packet loss, jitter, and end-to-end delay.

This research work is composed as follows; section 2 surveys several existing research papers based on VoIP over LTE network. In section 3, a brief explanation of an EVS-TCP Reno-VoIP method for better voice services is presented. In the section 4, the comparison of the experimental results of existing and EVS-TCP Reno-VoIP methods are presented. The conclusion is made in section 5 .

\section{Literature survey}

This section analyses some significant research approaches suggested by the researchers on VoIP over LTE network. The brief evaluation of some significant contributions to the existing methods is presented.

S. Ansari, and R. Gupta [11] proposed the performance of the voice over LTE networks using voice codec. In this work, the LTE provides a natural and unique QoS aware method. The LTE network provides a natural and unique QoS aware method to deliver better end-to-end facility. This research observed only the voice codec effects based on performance of end-to-end VoLTE. Here, a number of voice codec were taken in a different section modeled using the OPNET simulator. However, this method requires different voice codec for each block.

R. C. Soothar, M. Pathan, B. Qureshi, P. K. Butt, and G. Mujtaba [12] implemented the VoIP traffic service in the 4G LTE network by employing three parameters of the QoS, those are latency, throughput, and jitter by contracting the value with three voice codecs G.711, G.729 and EVS. In this work, the LTE was patched with NS2 that was not supported by default in the NS2. The EVS voice codec provided better performances in LTE compared to other voice code like G.711, G.729. The G.729 codec provided less throughput due to the low bit rate code compared to EVS and G.711 codec.

S. M. Abdullah, O. Younes, H. M. Mousa, and H. Abdul kader [13] presented the TCP algorithm to enhance the performance of the LTE network. The proposed method was controlling the receiver buffer size in the eNodeB. It was performed through partitioning the memory size of eNodeB among active users. This method avoided congestion in the buffer by controlling the receiver size of the TCP in the eNodeB. The congestion mechanisms created a dynamic change of window size of the TCP flow according to the less buffer size and number of users in the cell. This method has high delay and lose a lot of data.

A. Sengar [14] presented a comparison of LTE and WiMAX on fixed and mobile networks for TCP and UDP traffics using the NS2 simulator. In this paper, performance computation of the LTE and WiMAX were implemented on NS 2.34. Both the LTE and WiMAX were used in real-life scenarios to see how the various factors like distance, different modulation schemes, number of subscribers' stations, packet size affected the performance of LTE and WiMAX. The simulation was carried out for the user datagram protocol on fixed as well as mobile networks. The result obtained from the simulation showed that the performance metrics (throughput, delay and jitter) were affected due to the change in features like modulation scheme, number of nodes and distance between Base Station (BS) and Subscriber Section (SS).

The existing research analyzed jitter and latency of the VoIP in the third and fourth generation network by using the traffic measurement Jperf.

The measured traffic is compared to the major matric of Standard Quality Management (SQM). The measurements concentrated on the 2-QoS elements such as latency and jitter that significantly affect the quality of the network. To overcome this problem, the VoIP voice through WiMAX network quality is used and it satisfies better jitter and throughput by using proposed EVS-TCP Reno-VoIP method. In this research, four different types of the elements such as throughput, jitter, delay and packet lose computed to get an efficient connection to both $3 \mathrm{G}$ and $4 \mathrm{G}$ network.

\section{EVS-TCP-VoIP methodology}

This research discusses the voice quality characteristics of the EVS, the recently standardized the voice $3 \mathrm{GPP}$ codec. The EVS codec is used for improving the voice service and TCP Reno is used to avoid voice congestion in the LTE network. In addition to wideband and super wideband audio bandwidths, the EVS also supports narrowband speech having a sampling frequency of the $8 \mathrm{kHz}$ and full band speech having a sampling frequency of the $48 \mathrm{kHz}$ higher than that CDs. The EVS codec covers a wide range of the bit rates from 5.9 to 128 kbps. The TCP Reno is a variant of the TCP employed as a fast recovery technique, which allows the transmitter to avoid congestion the pipe and 
transfer the cwnd to cwnd/2 in space of a solitary Round Trip Time (RTT). Performance analysis can be done by NS2 and analyzing VoIP applications over the LTE network. The QoS of VoIP over LTE network and its performance via measurement of throughput, jitter, end-to-end delay and packet loss are evaluated over a variety of distances and network loads. The LTE network architecture is studied and then simulated. Then scenarios of single and multiple users are compared using VoIP over the LTE network to observe the effects on parameters.

\subsection{Long-term evolution architecture}

The architecture of the LTE network consists of 3- major parts such as User Equipment (UE), Evolved UMTS Terrestrial Radio Access Network (EUTRAN) and Evolved Packet Core (EPC). The UE segment contains a smart phone/mobile device. The evolved UMTS terrestrial radio access network segment a mobile device associated with BSs to provide radio connection for the user equipment. The EPC segment communicates with packet data in the outer world such as the internet or IP multimedia subsystem. In Fig. 1 shows the architecture of the LTE. The UE is directly employed by a user to communicate, that associates the evolved NodeB (eNodeB).

The EUTRAN associates every UE to LTE network. The EUTRAN connects NodeB that serves several cells in the LTE network, which sends and receives radio signals to UEs and performs certain tasks when a handover occurs. The eNodeB reduces the latency of all radio interface operations. It is interconnected via X2 as well as links with the EPC by the $\mathrm{S} 1$ interface. The architecture of the EUTRAN is shown in Fig. 2. The ECP segment consists of five significant nodes, those are packet data network (PDN), serving gateway, home subscriber station, and mobility management entity. The serving gateway serves as a router that transfer data packets between NodeB and users. It behaves as an anchor when the inter-eNodeB handover occurs.

The packet data network connects the LTE to the internet. The packet data network controls the services like traffic shaping, packet filtering and user charging policies, which is responsible for establishing, maintaining and deleting GPRS Tunneling Protocol (GTP) tunnels to the serving gateway. The home subscriber station is database, which has user information like user identification, user profile, and authentication. The mobility management entity manages the UE contexts like UE identity, security parameters, and mobile state. The architecture of the EPC is represented in Fig. 3.

\subsection{Enhanced voice service codec}

The quality of the speech is very important in the communication systems. The EVS is one of the speech coders standardized by the 3GPP for LTE that provides a data rate that ranges from 5.9 $128 \mathrm{kbps}$ with better voice services. The EVS codec supports audio bandwidth, provides high quality for mixed contents like music and speech/voice. Several codecs depend on the concept of the Algebraic Codec Excited Linear Prediction (ACELP) that may get affected by the changes in the spoken language. The EVS codec is performed with ACELP and Modified Discrete Cosine Transform (MDCT) coding technique. Fig. 4 shows the block diagram of the EVS encoder process.

In this research, the EVS codec is used to enhance the voice services. The EVS codec has 2operational modes: initial mode is the primary modes with 11-fixed bit rates such as $7.2 \mathrm{kbps}, 8$ kbps, $9.6 \mathrm{kbps}, 13.2 \mathrm{kbps}, 16.4 \mathrm{kbps}, 24.4 \mathrm{kbps}, 32$ kbps, 48 kbps, 64 kbps, 96 kbps and 128 kbps and

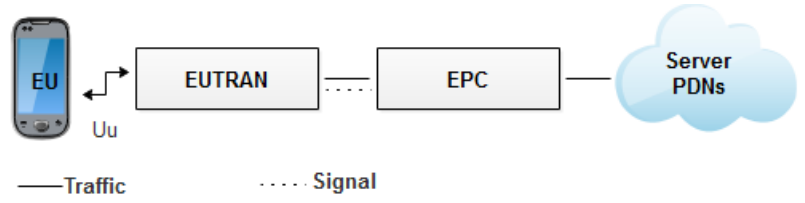

Figure. 1 The architecture of the LTE

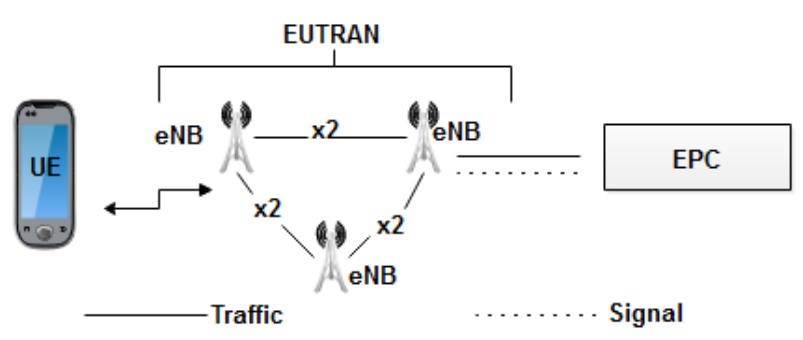

Figure. 2 The architecture of the EUTRAN

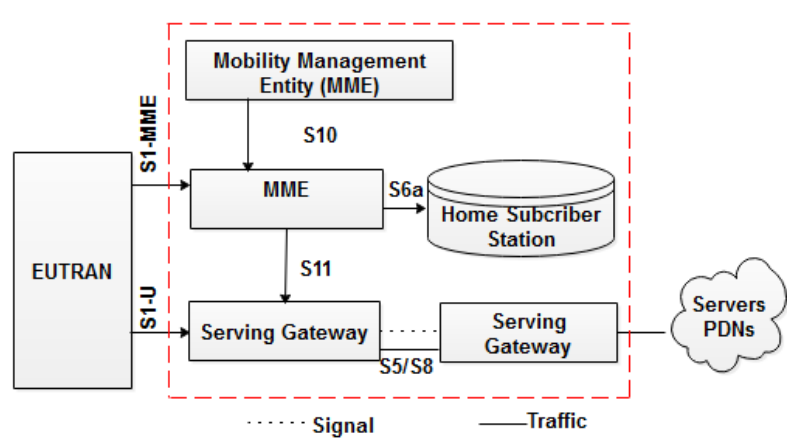

Figure. 3 The architecture of the packet data network 


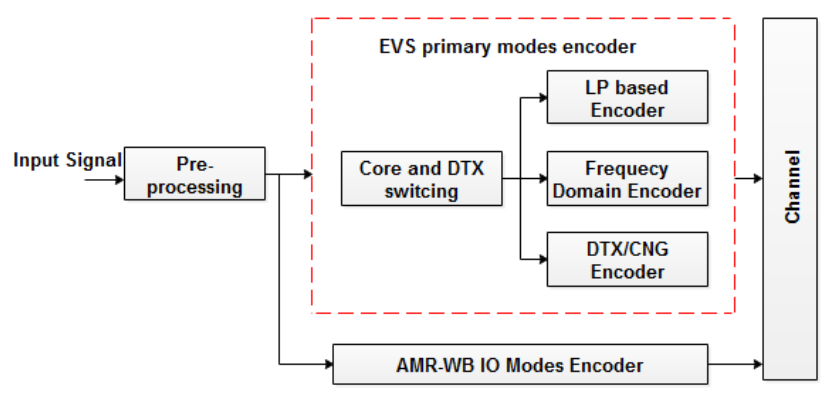

Figure. 4 Block diagram of the EVS encoder process

single variable bit such $5.9 \mathrm{kbps}$. The second mode is the EVS AMR-WB inter operable mode with 9 types of the bit rates such as $6.6 \mathrm{kbps}, 8.85 \mathrm{kbps}$, $12.65 \mathrm{kbps}, 14.25 \mathrm{kbps}, 15.85 \mathrm{kbps}, 18.25 \mathrm{kbps}$, $19.85 \mathrm{kbps}, 23.05 \mathrm{kbps}, 23.85 \mathrm{kbps}$. The EVS supports 4-sampling frequencies, those are $8 \mathrm{kHz}$ narrowband, $16 \mathrm{kHz}$ wideband, $32 \mathrm{kHz}$ super wideband and $48 \mathrm{kHz}$ full band. For an active signal, the EVS coder switches between linear predictive coding based on ACELP and frequency domain coding. In the case of inactive signal, Discontinuous Transmission (DTX)/ Comfort Noise Generation (CNG) coding is used to mitigate reduce bandwidth usage when periods of non-voice. The EVS decoder consists a jitter buffer management instead of preprocessing in the encoder.

The jitter buffer is a one of the shared data area, where voice packets can be composed, stored and sent to the voice processor in an evenly the spaced intervals. High-frequency elements of the input signal and low-frequency components are encoded with ACELP and this combination is again encoded with bandwidth extension methods where the fine structure of the higher spectrum is denoted partially with a low spectrum. In addition to the EVS primary mode and Adaptive Multi-Rate Wide Band (AMRWB) interoperable mode, a complete group of AMR-WB has bit stream decoded by existing AMRWB codec that is offered at the situations where the counterpart UE doesn't support EVS. The EVS decoder does not reverse the procedure taken at the encoder, but applies a new procedure to improve error resilience. The EVS codec produces high voice quality, packet error resilience, high compression efficiency compared to existing speech codecs. Further, quality enhancement is done by the introduction of super wideband and full band audio bandwidth.

\subsection{Transmission control protocol Reno}

In this paper, the TCP Reno is one of the variants of the TCP used for fast recovery method, which permits the transmitter to avoid congestion.
The TCP Reno follows the basic principle of the TCP Tahoe. The lost packets are easily detected by using TCP Reno technique. The congestion window and window threshold information are determined each and every time to send the accurate data to available bandwidth. This congestion control method is performed by considering the congestion packet loss information in network. Then the variety of transmission rates, transmission medium, routing path and status of the network change according to time. Fig. 5 shows the flow chart of TCP Reno congestion control. A slow start method is employed at the beginning of transmission. After receiving, each response Acknowledgement (ACK) segment, general formula is used to obtainCongestion Window(Cwnd)during slow start by employing Eq. (1).

$$
\text { Cwnd }=\text { Cwnd }+1
$$

The sender steps into congestion avoidance from a slow start when Cwnd exceedsSlow start threshold(Ssthresh). After receiving each ACK segment, Eq. (2) is employed to updateCwnd during congestion avoidance.

$$
\begin{gathered}
\text { Cwnd }=\text { Cwnd }+\frac{1}{\text { Cwnd }} \\
\text { ssthresh }=\frac{\text { Cwnd }}{2}, \text { Cwnd }=\text { ssthresh }+3 \\
\text { ssthresh }=\frac{\text { Cwnd }}{2}, \text { cwnd }=1
\end{gathered}
$$

The TCP sender employs the fast retransmit method to find repair loss based on the incoming duplicate ACKs. The fast retransmit method employs the arrival of three duplicate ACKs as an indication that a segment has been lost. After receiving three duplicate $\mathrm{ACKs}$, the TCP performs a retransmission of what appears to be missing segment and without waiting for the Retransmission Timer to Expire (RTO). When the third duplicate ACK is received, a set of Cwnd and Ssthresh values are given in the Eq. (3). Next, the fast retransmit method sends what appears to be missing segment. The fast recovery method governs the transmission of a new data until non-duplicate ACK arrives. The sender steps into congestion avoidance but not slow start again, which is computed by using Eq. (4).

\section{Experimental results}

The EVS-TCP Reno-VoIP method was implemented in the NS2 simulator. Table 1 shows 
the simulation parameters those are used in the EVS-TCP Reno VoIP methodology. The performance of the EVS-TCP Reno-VoIP method is measured in terms of the end-to-end delay, jitter, throughput and packet loss. The research work considered 160 nodes, which are randomly placed in the LTE network section. These nodes are deployed in the area of the $1000 \mathrm{~m} \times 1000 \mathrm{~m}$. User Datagram Protocol (UDP) is employed as a data agent.

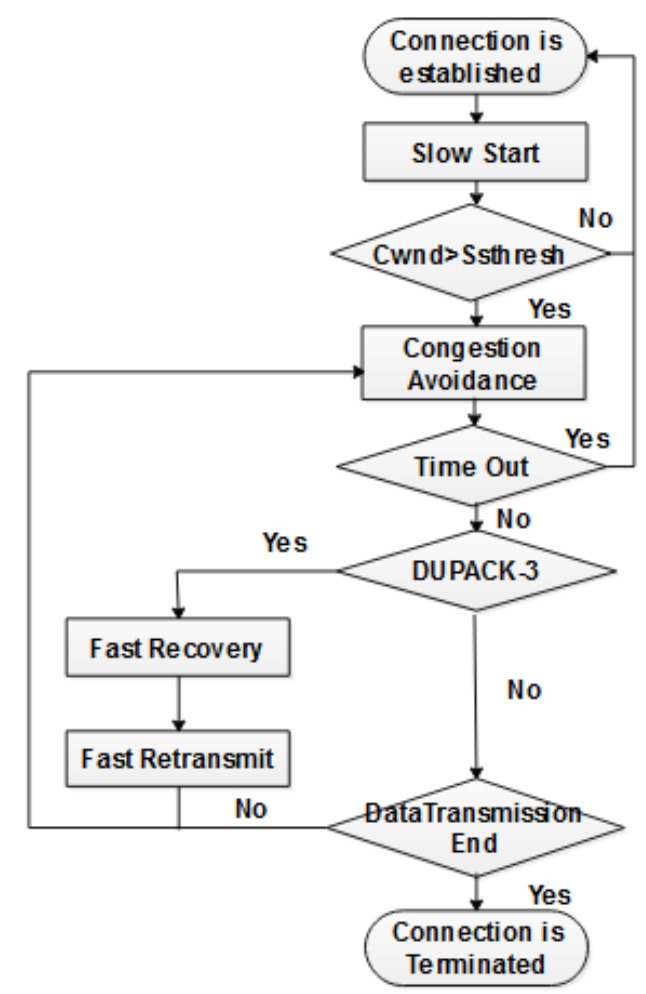

Figure. 5 Flowchart of the TCP Reno congestion control

Table 1. Simulation parameter

\begin{tabular}{|c|c|}
\hline Parameters & Value/Types \\
\hline Codec & EVS \\
\hline Traffic analysis & Jperf software \\
\hline Data analysis & Testbed experiments \\
\hline Traffic & TCP Reno \\
\hline Data Agent & $0.000 \mathrm{~ms}$ \\
\hline Simulation start time & $100.00 \mathrm{~ms}$ \\
\hline Simulation end time & 1024 bits \\
\hline Packet size & 1,2 \\
\hline $\begin{array}{c}\text { Number of mobile } \\
\text { nodes }\end{array}$ & $3,6,9$ \\
\hline Server nodes & $1,2,3$ \\
\hline eNB & $1000 \mathrm{~m} \times 1000 \mathrm{~m}$ \\
\hline Serving gateway & \\
\hline Network Area & \\
\hline
\end{tabular}

\subsection{Throughput}

Throughput computes how much amount of data is transferred from source to destination in a certain amount of time, which is commonly computed in Kbps. The throughput is computed by using Eq. (5)

$$
\text { Throughput }=\frac{\text { Packet Received }}{\text { Delay }}
$$

\subsection{Jitter}

Jitter is a variation in the packet latency. In other words, jitter measure the time variation in the packet inter-arrival time. When the packets are sent from transmitter side to receiver side, there is an end-toend delay variation between the packets. That time difference is called as jitter. Jitter is computed by using Eq. (6).

$$
\text { Jitter }=(T 4-T 3)-(T 2-T 1)
$$

Here, $T 2$ and $T 1$ represents the time of the leaving two consecutive packets from the source node and $T 4$ and $T 3$ denotes the time of arrival of two consecutive packets at the destination node.

\subsection{End-to-end delay}

End to end delay is represented as the reserved period of packets to be transferred between networks from sender to receiver. End to end delay is computed because the part of total delay within entire communication has associated with the quality of packets to offer receiver end nodes throughout the whole recursive run.

\subsection{Packet loss}

The packet loss is the percentage of the packets that lost during transmission from source to destination over the network. The packet loss is computed by using Eq. (7).

Packet loss $=\frac{\text { Packets Sent-Packets receive }}{\text { Packets sent }} \times 100$

This section provides the significances of the simulation results of the EVS-TCP-VoIP method. The EVS codec is used for analysis and execution of the VoIP traffic service in LTE network. Fig. 6 shows the comparison result of the throughput performance for existing and EVS-TCP Reno-VoIP methods. The performance of the EVS-TCP RenoVoIP method provides high throughput compared to existing methods such as EVS [12], G.711 [15] and 


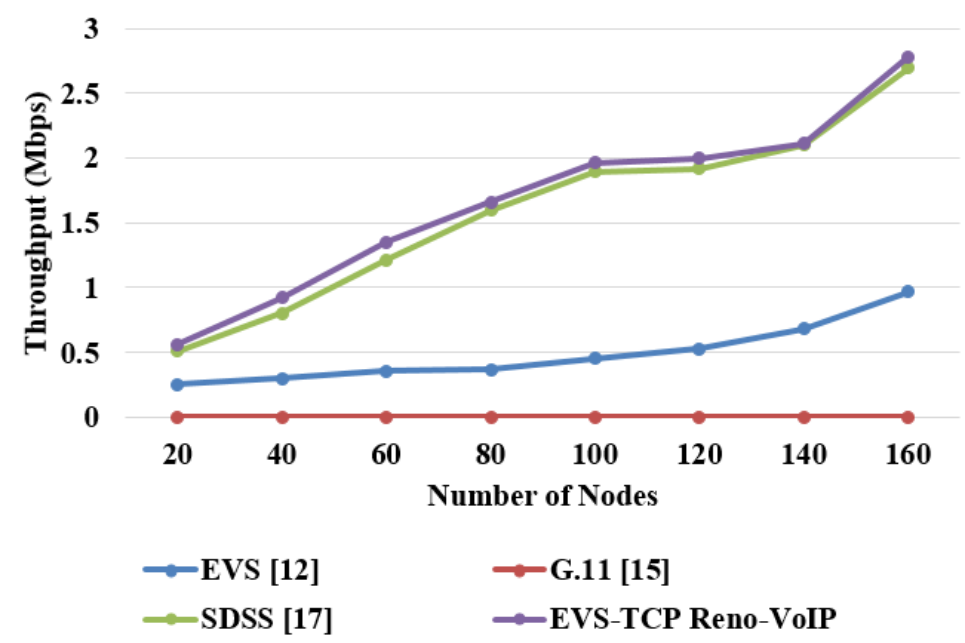

Figure. 6 Comparative analysis of throughput performance for an existing and proposed method

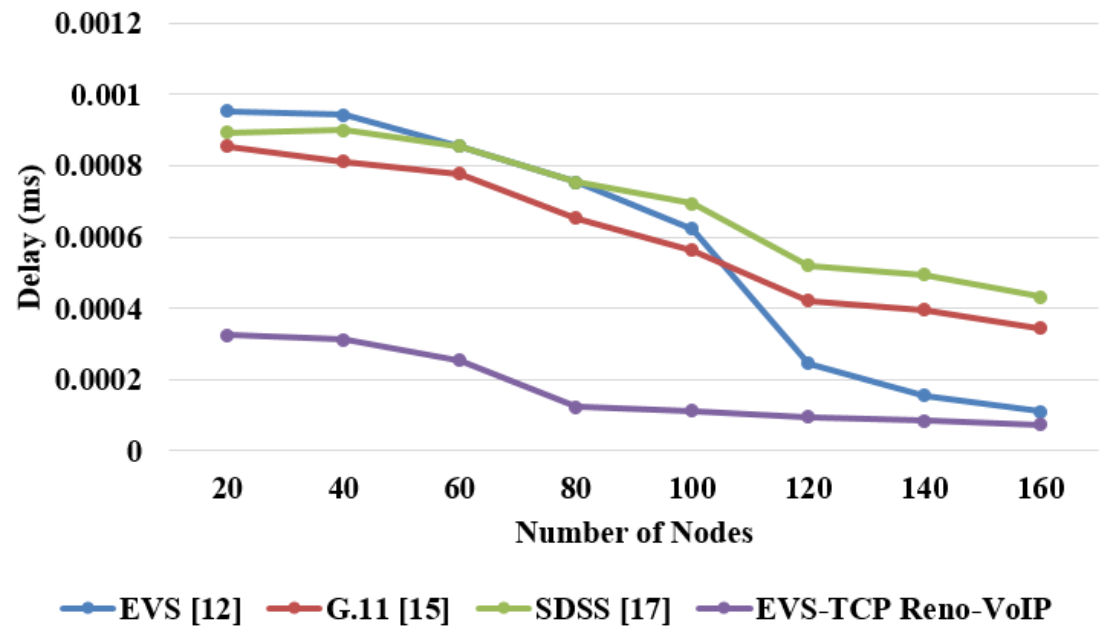

Figure. 7 Comparative analysis of delay performance for an existing and proposed method

SDSS [17]. In existing methods, the transmitter doesn't deliver packets properly due to congestion. So, the performance of throughput is not efficient. This comparison graph shows the different throughput level on one to one call. The proposed EVS-TCP Reno-VoIP method achieved 2.98 Mbps of throughput compared to existing methods. The given data efficiently transferred from source to destination by using EVS-TCP Reno-VoIP method at a particular time. The Comparison graph of the delay performance for existing and EVS-TCP RenoVoIP method is represented in Fig. 7. It is important to analyze the performance of delay of the LTE network because it directly degrades the quality of mobile services and application. This research analyzed the performance of delay of one to one communication in LTE network. This analysis is useful in computing delay performance of the LTE in a realistic environment. Furthermore, this research analysis will help to enhance the performance of delay of Next Generation Mobile
System (NGMS). In EVS-TCP Reno-VoIP method, the EVS codec has generated a lower data rate of delay compared to the existing method. Hence, from Fig. 7 is concluded that the performance of delay is reduced using EVS-TCP Reno-VoIP method on LTE network compared to the existing methods.

As shown in the comparison graph in fig.7, the delay is quite stable at $0.0001 \mathrm{~ms}$. Fig. 8 shows comparable performance of jitter of existing and EVS-TCP Reno-VoIP methods. In existing [12], [15] and [17], an increasing jitter shows that the packets arrive at varying delay at the receiver, it reflects adversely on the perceived quality. Here, every packet contains a voice sample sent from the sender. The packets that are not received with the same delay at the receiver end, the voice quality appears to be degraded. In this research, the proposed EVS-TCP Reno-VoIP method achieved less jitter compared to existing methods. This research employed row operation in excel to 

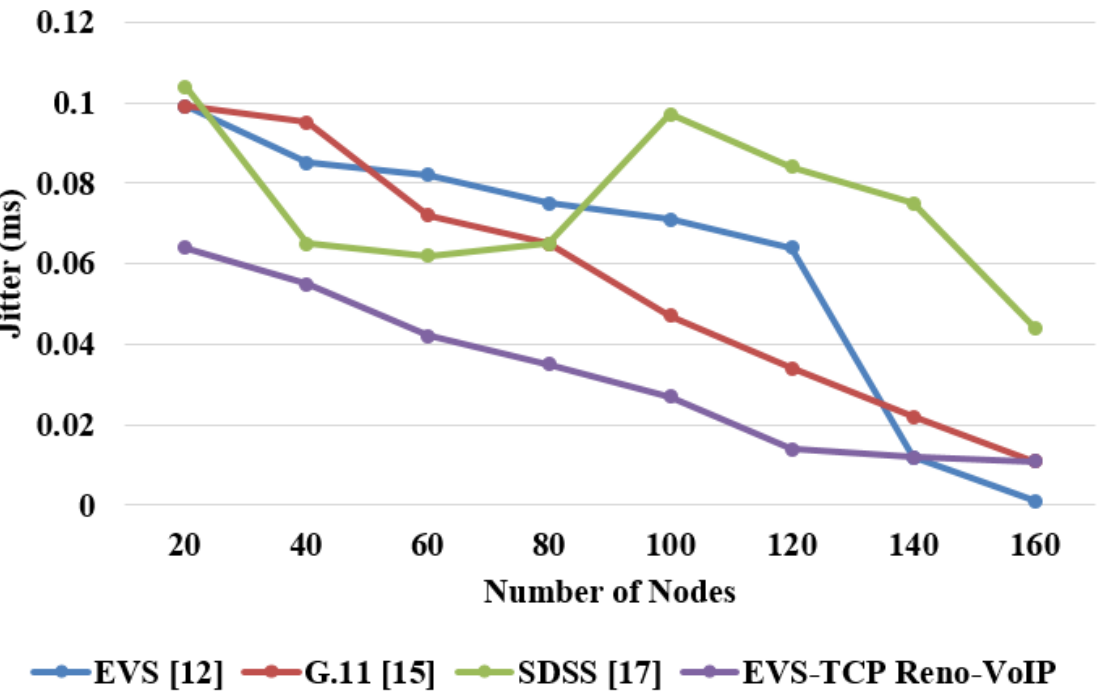

Figure. 8 Performance of jitter for an existing and proposed method

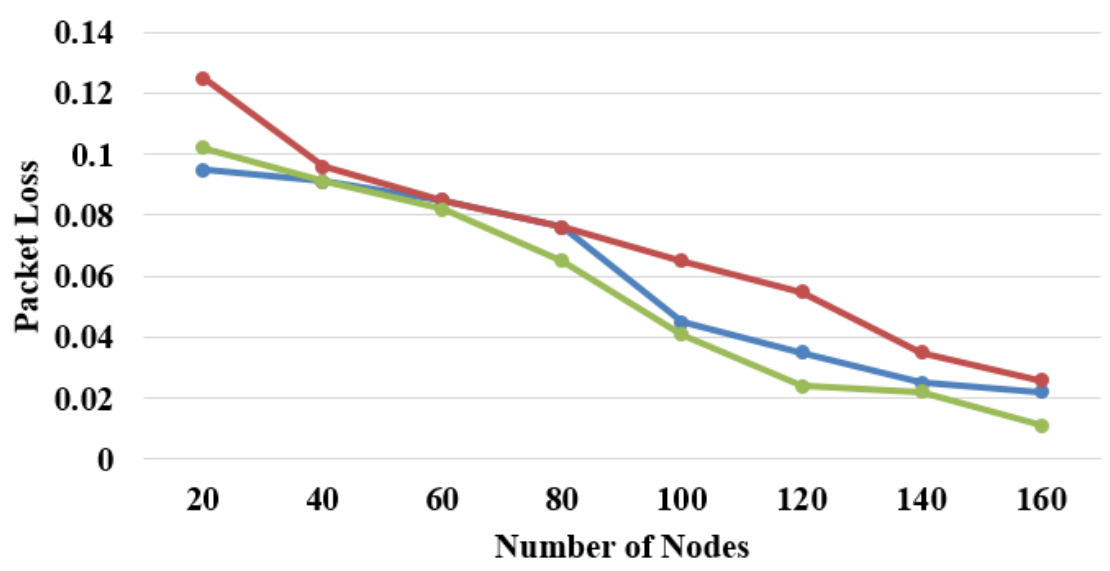

$\because$ CQA [16] $\multimap$ SDSS [17] $\multimap$ EVS-TCP Reno-VoIP

Figure. 9 Performance of packet loss for an existing and proposed method

compute jitter and plot the jitter graph. The obtained jitter value improved the performance of the overall system in terms tolerance and stability factor. Fig. 9 shows the performance of packet loss of existing and EVS-TCP Reno-VoIP method. The performance of the packet loss is computed based on the number of the packets sent on LTE cellular network that fail to reach their destination node. Packet loss is a major problem in the LTE cellular network due to the high-speed of traffic being identified in the network. In this research, EVS-TCP Reno-VoIP method achieved less packet loss by using the TCP Reno technique. The TCP Reno is able to identify up to $20 \mathrm{Mbps}$ line speed without packet loss.

In the Figs. 6, 7, 8, and 9, the comparative analysis is done between the proposed EVS-TCP Reno-VoIP method and existing methods EVS codec [12], G.723 [15], CQA [16] and SDSS [17]. Here, the EVS-TCP Reno-VoIP method is averagely improved $2.845 \%$ of the throughput, $0.0003 \%$ of the delay, $0.006 \%$ of the jitter and $0.011 \%$ of the packet loss compared to the existing methods. So, the proposed EVS-TCP Reno-VoIP method is much suitable for one to one communication process over the LTE network.

\section{Conclusion}

In this paper, the EVS-TCP Reno-VoIP method analyzed the performance of a VoIP on LTE network. The performance analysis was done by evaluating the QoS parameter of VoIP over LTE network. In this research, the EVS codec was employed for better voice services in the one-to-one communication. As well as the TCP Reno algorithm effectively avoided the voice congestion on the LTE network compared to the existing methods. The simulation results showed that the EVS-TCP RenoVoIP method increased the throughput and reduced delay, jitter and packet loss on the network. Hence, 
from the outcomes of the EVS-TCP Reno-VoIP method concluded that the EVS codec and TCP Reno are the most suitable algorithms for the one to one communication of VoIP on LTE network. The proposed EVS-TCP Reno-VoIP method can support both real and non-real time environmental applications. In the future work, the performance of the VoIP can be improved by using an efficient protocol with optimization techniques.

\section{References}

[1] K. Lau, G. C. Goodwin, T. Wigren, and L. Brus, "Improving the performance of cellular uplinks via power overbooking", IET Communications, Vol.11, No.9, pp.1512-1518, 2017.

[2] P. Sharma, "Evolution of mobile wireless communication networks- $1 \mathrm{G}$ to $5 \mathrm{G}$ as well as future prospective of next generation communication network", International Journal of Computer Science and Mobile Computing, Vol.2, No.8, pp.47-53, 2013.

[3] M. Kassim, R. A. Rahman, M. A. Aziz, A. Idris, and M. I. Yusof, "Performance analysis of VoIP over 3G and 4G LTE network", In: Proc. of International Conf. On Electrical, Electronics and System Engineering, pp.37-41, 2017.

[4] M. Rinne and O. Tirkkonen, "LTE, the radio technology path towards 4G", Computer Communications, Vol.33, No.16, pp.1894-1906, 2010.

[5] G. Epiphaniou, C. Maple, P. Sant, and G. A. Safdar, "Effects of iterative block ciphers on quality of experience for Internet Protocol Security enabled voice over IP calls", IET Information Security, Vol.6, No.3, pp.141-148, 2012.

[6] M. Z. Hasan and M. Z. Hussain, "Collective Study on Security Threats in VOIP Networks", International Journal of Scientific \& Technology Research, Vol.4, No.8, pp.20-23, 2015.

[7] U. P. D. Ani, "VoIP Security: Improving Quality of Service through the Analysis of Secured Transmission", International Journal of Computer Science and Business Informatics, Vol.15, No.1, 2015.

[8] Q. Ibrahim and N. Abdulghani, "Security enhancement of voice over Internet protocol using speaker recognition technique", IET Communications, Vol.6, No.6, pp.604-612, 2012.

[9] Y. Jung and C. Manzano, "Burst packet loss and enhanced packet loss-based quality model for mobile voice-over Internet protocol applications", IET Communications, Vol.8, No.1, pp.41-49, 2014.
[10] O. Çalik, U. Iricioğlu, G. K. Kurt, A. E. Pusane, A. S. Demiroglu, and G. Kayik, "Impact of retransmissions on the quality of experience in VoIP systems", In: Proc. of International Conf. On Electrical, Electronics and Biomedical Engineering, pp.617-621, 2016.

[11] S. Ansari and R. Gupta, "Evaluate Performance of Voice over LTE Networks using Voice Codec's", International Journal of Science, Engineering and Technology Research, Vol.5, No.5, 2016.

[12] R. C. Soothar, M. Pathan, B. Qureshi, P. K. Butt, and G. Mujtaba, "Analysis of VoIP Traffic Service in 4G LTE Cellular Networks", Indian Journal of Science and Technology, Vol.8, No.1, 2018.

[13] S. M. Abdullah, O. Younes, H. M. Mousa, and H. Abdul kader, "Enhancing Performance of TCP Variants in LTE", International Journal of Computer Applications, Vol.152, No.1, 2016.

[14] A. Sengar, "Comparison of LTE (Long Term Evolution) and WiMax on fixed and mobile networks for TCP, UDP traffics using NS2 Simulator", International Journal of Computer Science and Information Technologies, Vol.5, No.4, pp.5296-300, 2014.

[15] A. Agarwal and K. Agarwal, "The next generation mobile wireless cellular networks4G and beyond", American Journal of Electrical and Electronic Engineering, Vol.2, No.3, pp.92-97, 2014.

[16] N. M. Yusoff, D. M. Ali, and A. Idris, "Performance Analysis of Quality of Service and Energy Efficient Aware (QEEA) Scheduling Algorithm for Long Term Evolution (LTE)", Journal of Engineering Science and Technology, Vol.13, No.5, pp.1190-1204, 2018.

[17] Q. Liu and C.W. Chen, "Smart downlink scheduling for multimedia streaming over LTE networks with hard handoff', IEEE Transactions on Circuits and Systems for Video Technology, Vol.25, No.11, pp.1815-1829, 2015. 